\title{
Transparent biocompatible sensor patches for touch sensitive prosthetic limbs
}

\author{
Anindya Nag, Subhas Mukhopadhyay \\ Faculty of Science and Engineering \\ Macquarie University \\ Sydney, Australia
}

\author{
Jurgen Kosel \\ Sensing, Magnetism and Microsystems Group \\ King Abdullah University of Science and Technology \\ Saudi Arabia
}

\begin{abstract}
The paper presents the fabrication of transparent, flexible sensor patches developed using a casting technique with polydimethylsiloxane (PDMS) as substrate and a nanocomposite of carbon nanotubes (CNTs) and PDMS as interdigital electrodes. The electrodes act as strain sensitive capacitor. The prototypes were used as touch sensitive sensors attached to the limbs. Experiments results show the sensitivity of the patches towards tactile sensing. The results are very promising and can play a key role in the development of a cost efficient sensing system attached to prosthetic limbs.
\end{abstract}

\section{Keywords- PDMS; CNT; Nanocomposite; Prosthetic.}

\section{INTRODUCTION}

The advancement in medicine has taken a leap with the assistance of other fields of science in the last two decades $[1,2]$. Different kinds of diseases and ailments are being tackled in today's world which was practically impossible a few decades ago [3]. Sensing technology is one area which has been used substantially the medical field. The most common types of sensors are based on silicon substrates, due to the advantages of high electrical conductivity and resistance towards temperature and the precision and integration of the IC industry. While these sensors are working fine for applications like gas sensing [4, 5], phthalate detection [6], etc., but their association with monitoring of human physiological parameters is difficult, due to their rigidity and stiffness. Sensors with flexible substrates were introduced [7] to overcome these shortcomings of the silicon sensors. Light-weight, thinness, transparency and mechanical flexibility are some of the advantages of flexible sensors.

Different materials like PDMS [8], Polyethylene naphthalate (PEN) [9], Polyethylene terephthalate (PET) [10] have been used to develop flexible sensors. For the electrodes part, different kinds of nanomaterials [11] have developed as a substitute for their brittle counterpart electrodes used for rigid substrates. The fabrication of conductive nanocomposites for flexible sensor patches requires mixing the nanomaterial with the polymer used as a substrate for the sensor patch. The fabrication techniques also differ for different flexible sensors for different applications. Some of the common types like photolithography [12], screen printing [13], laser printing [14], etc. have been used.
Prosthesis has been used for a long time to replace a missing part of an amputee's body with an artificial one. The most common types of prosthesis done in the medical field is limb prostheses [15-17], where different organs of the upper or lower part of the body are joined to help in restoring a normal life to the person. The common materials used for fabricating prosthetic organs are fiberglass, nylon, Dacron, carbon, and Kevlar [18]. Control of stiffness and strength are some of the advantages of using these materials. But the brittle nature and the inability to remold the device once created are some of the common disadvantages associated with them. A material like Dacron in prosthetic devices can lead to the inability of the region to repair and grow due to the stiffness of the replaced part. This effect on the growth of tissue of the affected portion can lead to multiple replacements. In the case of Kevlar, it is difficult to shape the material into the form of the amputated organ unless operated with special tools and equipment. This increases the cost of the overall amputation process [19]. The material also corrodes in contact with chlorine. Thus, it is a state-ofart to develop sensors as a replacement of the commercially used devices.

Touch sensitive prosthetic limbs are an intriguing development in the sector of prosthesis where substantial work [20, 21] has been done over the past decade. Researchers have been trying to develop touch sensitive somatosensory organs which would send the sensory feedback to the amputee's brain. The devices have been tested so far on robots [22, 23] and artificially fabricated organs [24, 25]. An attempt was made [26] to develop brainmachine interfaced prosthetic limb. Higher-cost, complex testing systems, low response are some of the disadvantages of such systems.

In this paper, we report the fabrication of a novel transparent, nanocomposite-based flexible sensor patch which was used in the laboratory for tactile sensing. The sensor patch consists of a PDMS with strain sensitive interdigital nanocomposite electrodes. The electrodes were formed by mixing multi-walled carbon nanotubes (MWCNTs) and PDMS and formed by laser cutting.

The rest of the paper is organized as follows. Followed by the introduction given in section I, the working phenomenon of the electrodes is explained in section II. Section III gives the fabrication details with the characterization, and 
experimental results are given in section IV and V respectively. The conclusions are given in the final section.

\section{WORKING PRINCIPLE FOR FABRICATED ELECTRODES}

Interdigital electrode sensors are based on the principle of a parallel plate capacitor. The capacitance is a function of the distance between the electrodes; hence, it is sensitive to mechanical strain. The coplanar structure of the electrodes is well suited for non-invasive, one-sided measurements. Two electrodes, positive and negative with the inter-electrode distance (d) and pitch (p) are shown in figure 1. When an alternating voltage is supplied to the excitation electrode at a definite frequency, a potential difference is created between the two electrodes leading to dipole formation on the two electrodes creating an electric field to flow between them. Due to the planar structure of the sensor, the field bulges from one electrode to another. If a material is in proximity to the electrodes, the electric field will penetrate through the material, leading to a measurable impedance change. The phenomenon is depicted in figure 2. Materials like meat [27], beverages [28], milk [29] have been studied previously with this types of sensing concept. The developed sensor patch is strain sensitive in nature where the capacitance changes as a function of the pitch on the application of strain. Figure 3 shows the working phenomenon.

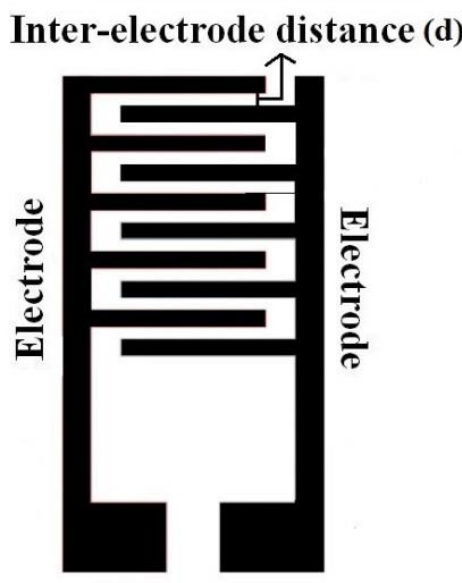

Fig. 1: Schematic diagram of the interdigital sensor [30].

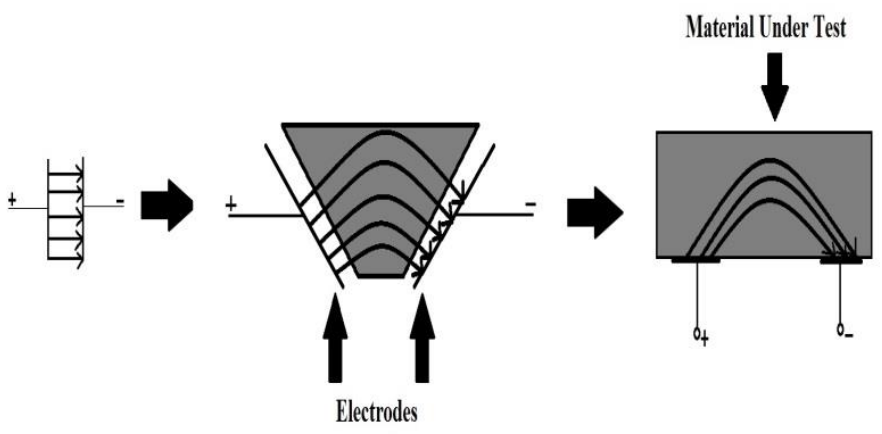

Fig. 2: Principle of interdigital electrodes.

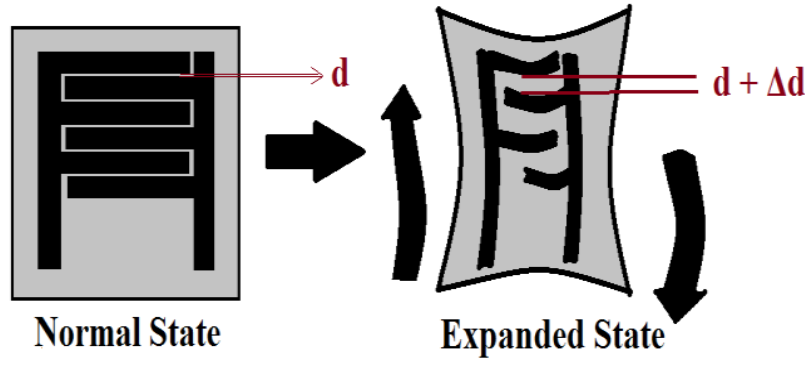

Fig. 3: Working principle of the developed strain sensitive patch

\section{FABRICATION PROCESS}

The fabrication of the sensor patches was done by a casting method. This technique has been used [31] due to its certain advantages like low-cost, constructing thin and flexible devices and high production rate. PDMS was used as the substrate material. This organic polymer was chosen because for its high transparency, low cost, low Young's modulus and high resistance towards temperature [32]. For the electrode part, multi-walled carbon nanotubes (MWCNTs) were used to form a nanocomposite with the PDMS. Carbon nanotubes are $\mathrm{sp}^{2}$ hybridized carbon atoms bundled into sheets. High electrical conductivity, high flexibility, light weight and high tensile strength are some of the advantages of carbon nanotubes [33]. Carbon nanotubes are available in two forms, single-walled and multi-walled. Here MWCNTs are preferred over single-walled ones due to higher conductivity, mechanical stability and resistance towards bond breakage when mixed with polymers [34]. Functionalized MWCNTs were chosen for this work due to their advantage of better dispersion in the polymer compared to the un-functionalized MWCNTs [35]. The functionalization group used for the nanotubes is carboxylic acid (-COOH) to increase the conductivity of the resultant nanotubes.

Figure 2 shows the schematic diagram of the fabrication steps of the sensor patches. Poly (methyl methacrylate) (PMMA) was used as the template for casting purposes. A $\mathrm{CO}_{2}$ laser (Universal Laser Systems) was used to create trenches on the template in the form of interdigitated electrodes. The trenches created on the PMMA template were 500 microns deep. The nanocomposite was formed by mixing $4 \%$ wt. of MWCNTs into PDMS. This weight was optimized after a series of experiments on different weight values to have a balance between the flexibility and conductivity of the developed nanocomposite. Figure 5 is an SEM image of the nanocomposite. The black regions in the image are the carbons nanotubes while the corresponding black regions are the PDMS. The white regions shown in the picture are the PDMS, and the contemporary black regions are the MWCNTs. The nanocomposite was cast onto PMMA template. The height of the cast nanocomposite was made uniform to 500 microns using a casting knife (SHEEN, 1117/1000 mm). The cast nanocomposite was cured in an oven at $80^{\circ} \mathrm{C}$ for 8 hours. This was followed by casting of PDMS (SYLGARD ® 184, Silicon Elastomer 
Base) at a ratio of 10:1 of base elastomer (pre-polymer) and curing agent (cross-linker) over the cured nanocomposite on the template.

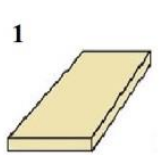

PMMA template

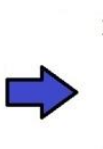

2
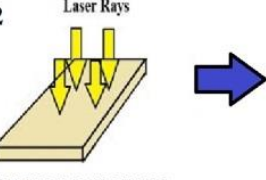

Laser ray exposure

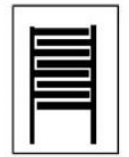

Sensor patch peeled off from the template

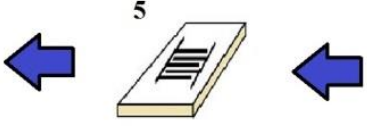

PDMS substrate casted

on the cured

nanocomposite electrodes

Fig. 4(1): PMMA template taken for trenching 2(2): Laser exposure done on the template. 2(3) Cavities formed on the template. 2(4):

Nanocomposite cast on the trenches on PMMA template. 2(5): PDMS cast on top of cured nanocomposite. 2(6): PDMS peeled off to form the sensor patch.

The height was adjusted to $1 \mathrm{~mm}$ by the casting knife. A curing step followed again in an oven at $80^{\circ} \mathrm{C}$ for 8 hours. The final sample was peeled off carefully from the template yielding the sensor patch as shown in figure 6(a). The black spots outside the electrodes are residual nanocomposites remaining on the PMMA template when the nanocomposite layer on the trenches where made uniform. The width of the electrodes was 600 microns, and the distance between two consecutive electrodes was 300 microns. Figure 6(b) shows a sensor patch attached to the hand. The sensor patch was sticking to the hand without any adherent due to the sticky nature of the PDMS.

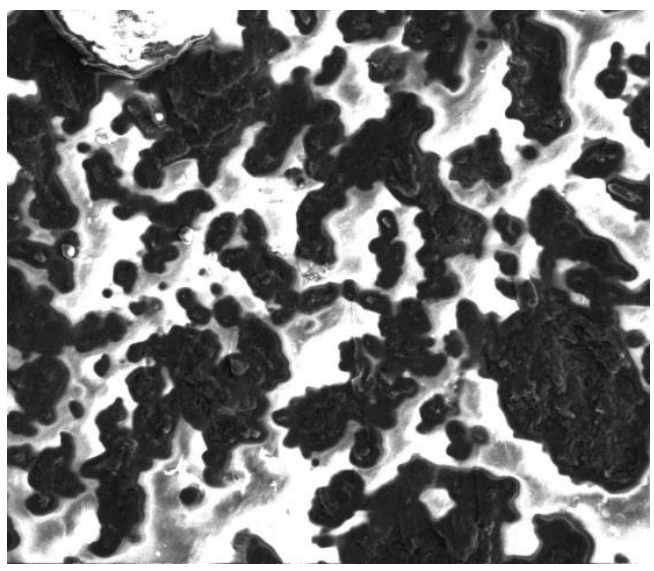

Fig. 5: SEM image of the CNT/PDMS nanocomposite.

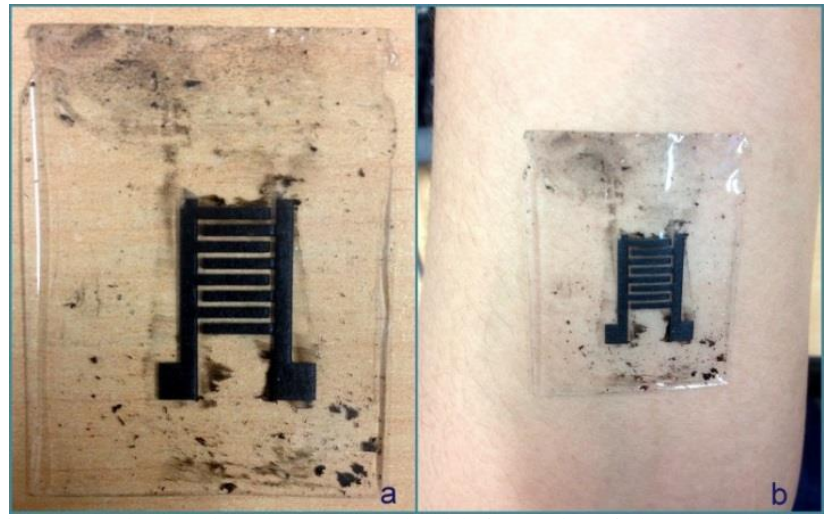

Fig. 6: (a) The developed sensor patch with four pairs of electrodes having a width of 200 microns of each electrode and 150 microns between two consecutive electrodes (b) Attachment of the sensor on hand.

\section{CharacterizATION OF THE SENSOR PATCH}

Testing of the sensor patches was done using a HIOKI 353250 LCR Hi-TESTER using Kelvin probes to connect the patch with the device. The frequency was swept between 350 $\mathrm{Hz}$, and $1 \mathrm{MHz}$ was done to determine the optimum frequency for operation. Figure 7 shows the change in phase angle of the sensor patch impedance over the frequency. The negative values of the phase angle represent the capacitive behavior of the patch. The change in phase angle is highest at $2736 \mathrm{~Hz}$ between 0 and $-90^{\circ}$ for the tested patch which makes it optimum frequency for the sensor patch. This frequency was fixed during the experimental operation.

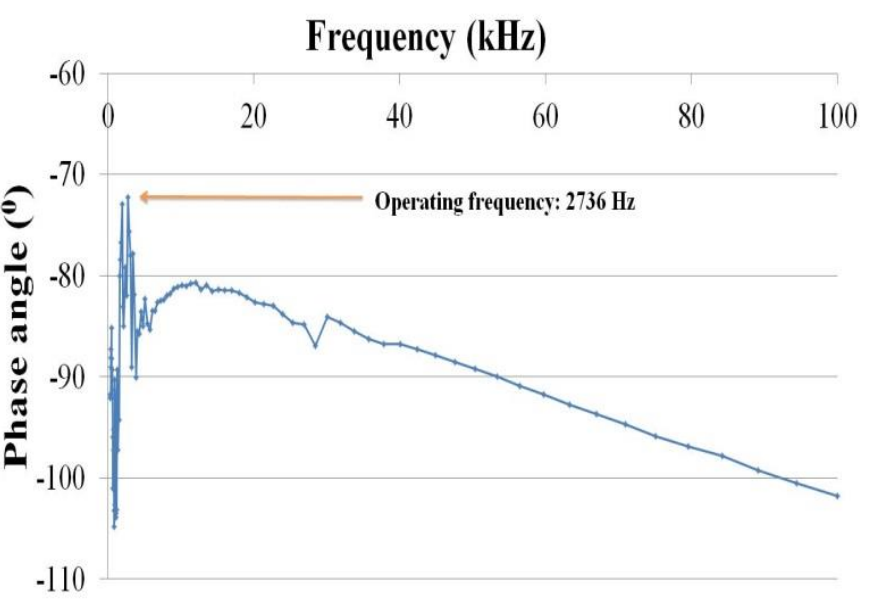

Fig. 7: Response of the sensor patch regarding phase angle with the change in frequency.

\section{EXPERIMENTAL RESULTS}

The sensing performance of the sensor patch was evaluated by using it as a tactile sensor at the tip of the forefinger. The patch was connected to the LCR meter via Kelvin probes as shown in figure 8 . An alternating voltage of $1 \mathrm{~V}$ peak-topeak was given as an input to the connected sensor patch. 


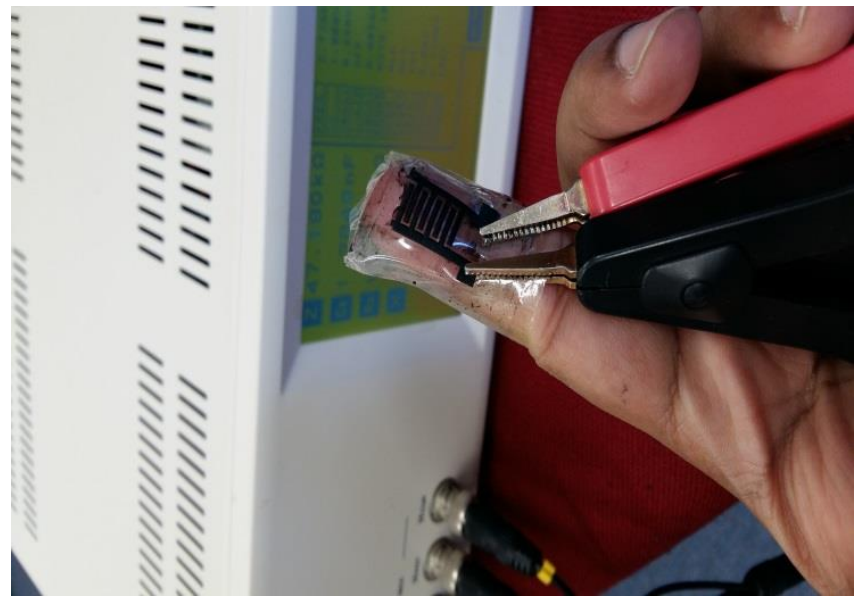

Fig. 8: The sensor patch attached to the fingertip and connected to the LCR meter for tactile sensing.

A tactile signal was obtained by analyzing the change in capacitance in $\mathrm{pF}$ with respect to time. Figures 9-13 show the sensor response for two conditions; touch and no touch. The results are represented in the change of absolute capacitance against time. Touch refers to the situation when the sensor attached to the finger is pressed against a plane surface. No touch refers to the situation when the finger is free from any contact. The results differ in the range of capacitance in touched condition due to the application of different pressures with the finger on the object. As seen from the results, the sensor patch responds well to the two defined conditions and the touched condition reaches higher values distinguishing the no-touch condition. The change in capacitance with different weights is shown in figure 14 . Different weights were kept on the sensing area of the patch to analyze the change in capacitance with weight. It is seen from the graph that the readings change linearly with the weights.

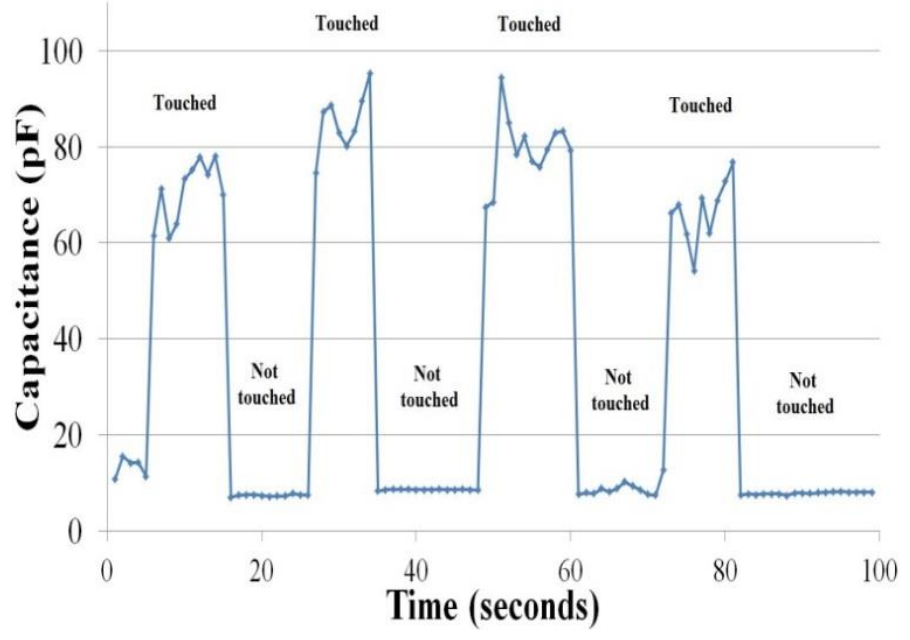

Fig. 9: Tactile sensing response of the sensor patch for a pressure of 42.2 $\mu \mathrm{Pa}$

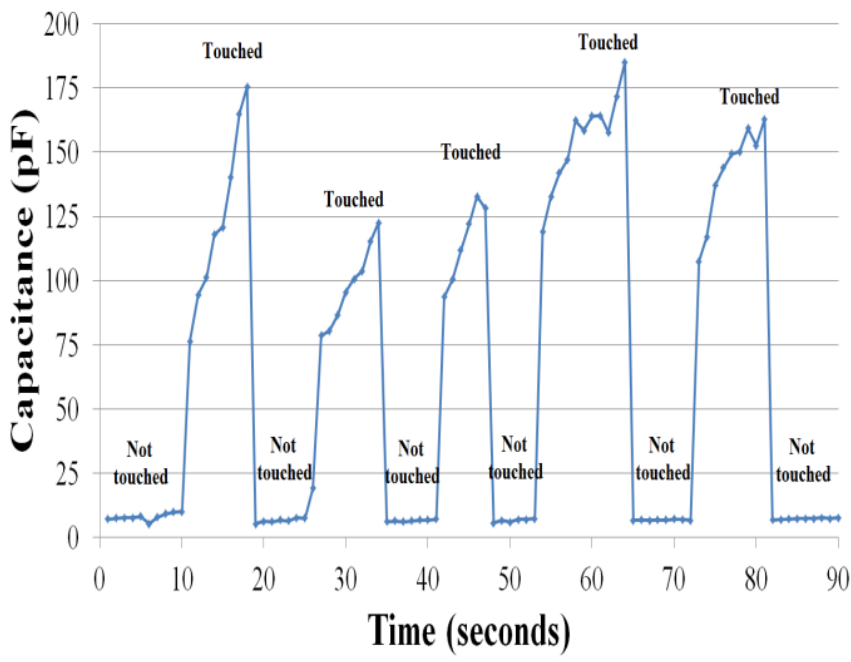

Fig. 10: Tactile sensing response of the sensor patch for a pressure of 54.6 $\mu \mathrm{Pa}$

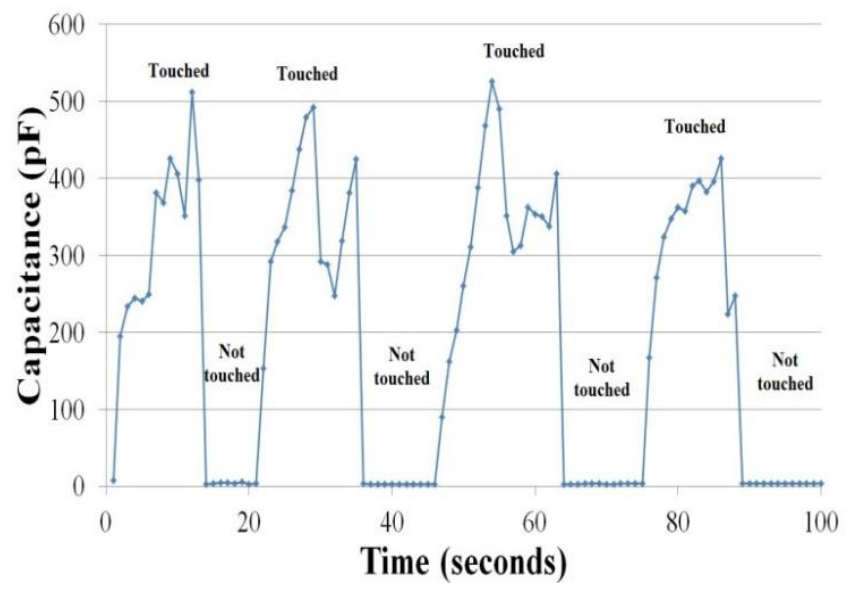

Fig. 11: Tactile sensing response of the sensor patch for a pressure of 68.2 $\mu \mathrm{Pa}$

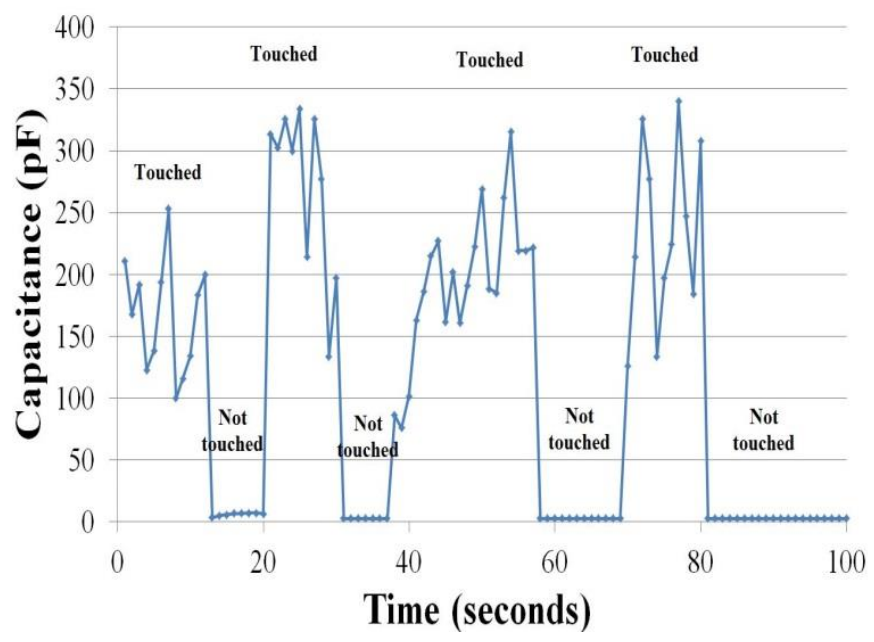

Fig. 12: Tactile sensing response of the sensor patch for a pressure of 62.4 $\mu \mathrm{Pa}$ 


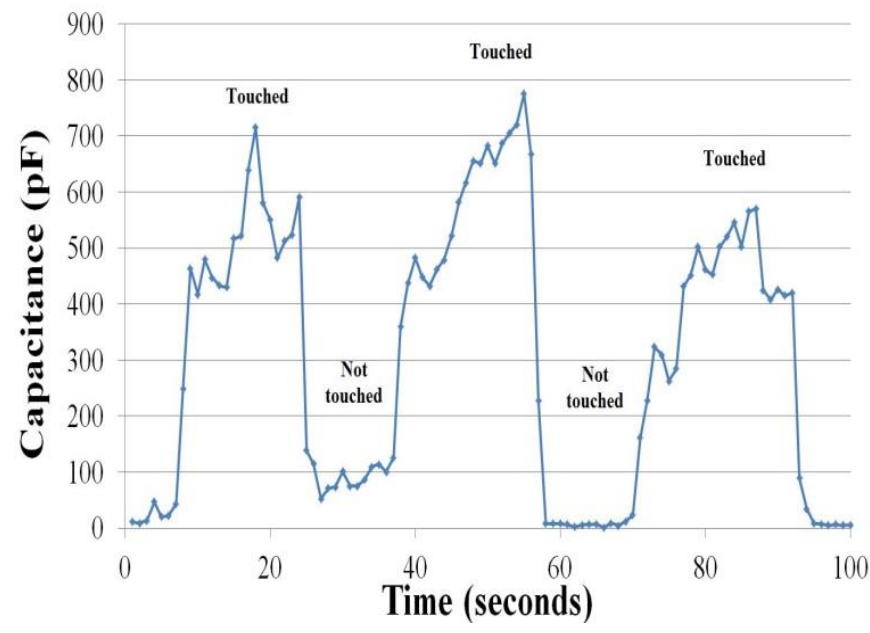

Fig. 13: Tactile sensing response of the sensor patch

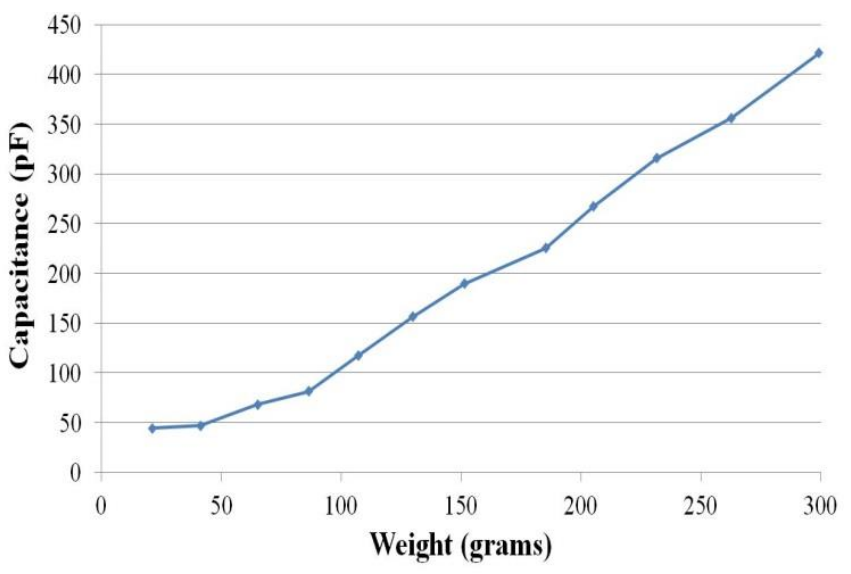

Fig. 14: Variation of capacitance on different weight values

Some glitches can be observed during the touched condition, which might be related to the experimental setup, where it is not possible to apply a constant pressure with the finger to the object. Also, since the sensor patch was connected with tapes at the tip of the finger, the tapes might come loose on bending the patch which could be a reason for the sudden change in capacitance. The capacitance in no touch condition has some non-zero values. This is due to the minimum strain on the sensor patch caused due to its bending around the finger.

\section{CONCLUSION}

The fabrication of transparent, flexible and cheap sensor patches was shown in the paper which was used as a touch sensitive patch. The obtained results are very satisfactory and could be used on to extend the work behind this idea. This could help to reduce the fabrication cost and equip prosthetic organs with efficient tactile sensors. The next step would be to use the sensor prototypes to validate their functionality and to include them in an embedded system would also be an option to develop a complete prosthetic system for human sensing.

\section{ACKNOWLEDGEMENT}

The authors would like to thank King Abdullah University of Science and Technology (KAUST), Saudi Arabia to let them use their research facilities to design and develop the sensor prototypes. They would also acknowledge Massey University, New Zealand to give opportunities to characterize and test the fabricated prototypes.

\section{REFERENCES}

F. Zhang, S. A. Hackworth, X. Liu, H. Chen, R. J. Sclabassi, and M. Sun, "Wireless energy transfer platform for medical sensors and implantable devices," in Engineering in Medicine and Biology Society, 2009. EMBC 2009. Annual International Conference of the IEEE, 2009, pp. 1045-1048.

A. M. Al-Busaidi and L. Khriji, "Wearable wireless medical sensors toward standards, safety and intelligence: a review," International Journal of Biomedical Engineering and Technology, vol. 14, pp. 119-147, 2014.

J. C. Carey, "Significance of case reports in the advancement of medical scientific knowledge," American Journal of Medical Genetics Part A, vol. 140, pp. 2131-2134, 2006

S. Mukhopadhyay, A. Nag, A. Zia, X. Li, and J. Kosel, "Novel sensing approach for LPG leakage detection: Part I: Operating Mechanism and Preliminary Results," 2015.

S. Mukhopadhyay, A. Nag, A. Zia, X. Li, and J. Kosel, "Novel sensing approach for LPG leakage detection: Part II: Effects of particle size, composition and coating layer thickness," 2015.

A. I. Zia, N. Afsarimanesh, L. Xie, A. Nag, I. Al-Bahadly, P. $\mathrm{Yu}$, et al., "Improved detection limits for phthalates by selective solid-phase micro-extraction," in 2015 9th International Conference on Sensing Technology (ICST), 2015, pp. 733-738.

A. Alfadhel and J. Kosel, "Magnetic nanocomposite cilia tactile sensor," Advanced Materials, vol. 27, pp. 7888-7892, 2015.

A. Mata, A. J. Fleischman, and S. Roy, "Characterization of polydimethylsiloxane (PDMS) properties for biomedical micro/nanosystems," Biomedical microdevices, vol. 7, pp. 281293, 2005.

R. Shabannia and H. Abu-Hassan, "Vertically aligned $\mathrm{ZnO}$ nanorods synthesized using chemical bath deposition method on seed-layer $\mathrm{ZnO} /$ polyethylene naphthalate (PEN) substrates," Materials Letters, vol. 90, pp. 156-158, 2013.

L. Wang, J. Luo, J. Yin, H. Zhang, J. Wu, X. Shi, et al., "Flexible chemiresistor sensors: thin film assemblies of nanoparticles on a polyethylene terephthalate substrate," Journal of Materials Chemistry, vol. 20, pp. 907-915, 2010.

M. Segev-Bar and H. Haick, "Flexible sensors based on nanoparticles," ACS nano, vol. 7, pp. 8366-8378, 2013.

A. Nag, A. I. Zia, S. Mukhopadhyay, and J. Kosel, "Performance enhancement of electronic sensor through maskless lithography," in 2015 9th International Conference on Sensing Technology (ICST), 2015, pp. 374-379.

S. Ito, P. Chen, P. Comte, M. K. Nazeeruddin, P. Liska, P. Pechy, et al., "Fabrication of screen - printing pastes from $\mathrm{TiO} 2$ powders for dye - sensitised solar cells," Progress in photovoltaics: research and applications, vol. 15, pp. 603-612, 2007.

[14] J. Barron, P. Wu, H. Ladouceur, and B. Ringeisen, "Biological laser printing: a novel technique for creating heterogeneous 3 dimensional cell patterns," Biomedical microdevices, vol. 6, pp. 139-147, 2004.

[15] P. Erik Scheme MSc and P. Kevin Englehart PhD, "Electromyogram pattern recognition for control of powered upper-limb prostheses: State of the art and challenges for clinical use," Journal of rehabilitation research and development, vol. 48, p. 643, 2011.

[16] A. Muzumdar, Powered Upper Limb Prostheses: Control, Implementation and Clinical Application; 11 Tables: Springer Science \& Business Media, 2004. 
[17] A. Fougner, Ø. Stavdahl, P. J. Kyberd, Y. G. Losier, and P. A. Parker, "Control of upper limb prostheses: terminology and proportional myoelectric control-a review," Neural Systems and Rehabilitation Engineering, IEEE Transactions on, vol. 20, pp. 663-677, 2012

[18] Materials used for prosthetic limbs. Available: http://www.amputee-

coalition.org/inmotion/sep oct 98/matinprs.html. Last accessed on 09.08.2016

[19] What is Kelvar? Available: http://www.technologystudent.com/joints/kevlar2.html. Last accessed on 09.08.2016.

[20] M. C. Carrozza, P. Dario, F. Vecchi, S. Roccella, M. Zecca, and E. Sebastiani, "The CyberHand: on the design of a cybernetic prosthetic hand intended to be interfaced to the peripheral nervous system," in Intelligent Robots and Systems, 2003.(IROS 2003). Proceedings. 2003 IEEE/RSJ International Conference on, 2003, pp. 2642-2647.

[21] J. J. Boland, "Flexible electronics: Within touch of artificial skin," Nature materials, vol. 9, pp. 790-792, 2010.

[22] J. Dargahi, "A piezoelectric tactile sensor with three sensing elements for robotic, endoscopic and prosthetic applications," Sensors and Actuators A: Physical, vol. 80, pp. 23-30, 2000.

[23] G. Cannata, M. Maggiali, G. Metta, and G. Sandini, "An embedded artificial skin for humanoid robots," in Multisensor Fusion and Integration for Intelligent Systems, 2008. MFI 2008. IEEE International Conference on, 2008, pp. 434-438.

[24] A. Trampuz, J. M. Steckelberg, D. R. Osmon, F. R. Cockerill Iii, A. D. Hanssen, and R. Patel, "Advances in the laboratory diagnosis of prosthetic joint infection," Reviews in Medical Microbiology, vol. 14, pp. 1-14, 2003.

[25] W. Zimmerli, "Prosthetic-joint-associated infections," Best Practice \& Research Clinical Rheumatology, vol. 20, pp. 10451063, 2006.
(2013). Touch-Sensitive Prosthetic Limbs Take Step Forward in Monkey Study. Available: http://www.livescience.com/40405 touch-sensitive-prosthetic-limbs-monkey-study.html. Last accessed on 09.08.2016.

[27] S. C. Mukhopadhyay and C. P. Gooneratne, "A novel planartype biosensor for noninvasive meat inspection," Sensors Journal, IEEE, vol. 7, pp. 1340-1346, 2007.

[28] A. I. Zia, M. S. A. Rahman, S. C. Mukhopadhyay, P.-L. Yu, I. H. Al-Bahadly, C. P. Gooneratne, et al., "Technique for rapid detection of phthalates in water and beverages," Journal of Food Engineering, vol. 116, pp. 515-523, 2013.

[29] S. C. Mukhopadhyay, C. P. Gooneratne, G. S. Gupta, and S. Demidenko, "A low-cost sensing system for quality monitoring of dairy products," Instrumentation and Measurement, IEEE Transactions on, vol. 55, pp. 1331-1338, 2006.

[30] H. Bai and G. Shi, "Gas sensors based on conducting polymers," Sensors, vol. 7, pp. 267-307, 2007.

[31] W. S. Wong and A. Salleo, Flexible electronics: materials and applications vol. 11: Springer Science \& Business Media, 2009.

[32] PDMS: A review Available: http://www.elveflow.com/microfluidic-tutorials/microfluidicreviews-and-tutorials/the-poly-di-methyl-siloxane-pdms-andmicrofluidics/. Last accessed on 09.08.2016.

[33] (Course: Information Technology). Available: http://pvaughn.blogspot.co.nz/2011/02/carbon-nanotubesadvantages-and.html Last accessed on 09.08.2016.

[34] R. E. Smalley, M. S. Dresselhaus, G. Dresselhaus, and P Avouris, Carbon nanotubes: synthesis, structure, properties, and applications vol. 80: Springer Science \& Business Media, 2003.

[35] A. Eatemadi, H. Daraee, H. Karimkhanloo, M. Kouhi, N Zarghami, A. Akbarzadeh, et al., "Carbon nanotubes: properties, synthesis, purification, and medical applications," Nanoscale Res Lett, vol. 9, p. 393, 2014. 